\title{
LISTEN TO YOUR DRIVE: SONIFICATION ARCHITECTURE AND STRATEGIES FOR DRIVER STATE AND PERFORMANCE
}

\author{
Steven Landry ${ }^{1}$, David Tascarella ${ }^{2}$, Myounghoon Jeon ${ }^{12}$, \& S. Maryam FakhrHosseini ${ }^{1}$ \\ Mind Music Machine Lab \\ ${ }^{1}$ Department of Cognitive and Learning Sciences \\ ${ }^{2}$ Department of Computer Science \\ Michigan Technological University \\ 1400 Townsend Drive, Houghton, MI 49931, USA \\ \{sglandry, datascar, mjeon, sfakhrho\}@mtu.edu
}

\begin{abstract}
Driving is mainly a visual task, leaving other sensory channels open for additional information communication. As the level of automation increases in vehicles, monitoring the state and performance of the driver and vehicle shifts from the secondary to primary task. Auditory channels provide the flexibility to display a wide variety of information to the driver without increasing the workload of driving task. It is important to identify types of auditory displays and sonification strategies that provide integral information necessary for the driving task, and not overload the driver with unnecessary or intrusive data. To this end, we have developed an in-vehicle interactive sonification system using the medium-fidelity simulator and neurophysiological devices. The system is intended to integrate driving performance data and driver affective state data in real-time. The present paper introduces the architecture of our invehicle interactive sonification system and potential sonification strategies for providing feedback to the driver in an intuitive and non-intrusive manner.
\end{abstract}

\section{INTRODUCTION}

Automobiles and in-vehicle safety systems have been improved over the past several decades [1]. Researchers classify driving safety strategies into two categories: Passive and Active/Primary safety applications. Passive strategies, such as airbags, help people stay alive and uninjured in an accident [1], while active strategies, such as collision warnings and electronic stability control, aid in the prevention of car crashes and improve drivers' performance [2-3]. These active strategies reduce drivers' errors and improve their performance especially when they are engaged in secondary or tertiary tasks.

Since the human visual system is mostly busy with the driving task, designing active safety systems based on other sensory channels (e.g., auditory modality) is recommended. Auditory channels provide the flexibility to display a wide variety of information to the driver continuously. According to multiple resource theory [4], each task has a vector that shows the number and qualitative level of the attentional

\section{(c) (1) (9)} Attribution - Non Commercial 4.0 International License.

The full terms of the License are available at

http://creativecommons.org/licenses/by-nc/4.0/ resources. Assuming that driving includes visual, spatial, and manual resources, the amount of load within each resource depends on the task demands. Therefore, it is important to identify the appropriate auditory displays and sonification strategies that can provide integral information necessary for the driving task, and not overload the driver with unnecessary or intrusive data.

Sonification is a method for presenting data with sound [5]. The idea behind it is that people can perceive changes and draw conclusions easier and faster by listening to acoustic data in real time. A previous study [6] suggested that sonifying aggressive drivers' emotion will help them improve their driving performance in two ways: regulating their affect and providing appropriate feedback. This retrospective method can be used to return angry drivers' mood to a safer neutral mood. In contrast, in the present paper, we attempt to sonify drivers' performance and affective states in a more proactive way. Thus, this real-time sonification can also make a feedforward loop in terms of driving behavior and road safety.

\section{DATA ROUTING FOR DRIVING PERFORMANCE AND DRIVER STATE}

\subsection{Data routing software from the driving simulator}

As our driving sonification research platform, we use a medium-fidelity driving simulator, NADS (National Advanced Driving Simulator) MiniSim (Figure 1). Since the simulator provides all the driving data afterwards, the first step to sonify driving behavior in real-time was to develop a program that can relay the driving data in real-time. The first stage of our data routing software initializes the majority of the programs and lets the user set up the session. This includes selecting the driving variables (e.g., speed, pedal force, lane deviation, steering angle, etc.) of interest that our program will extract, display, record, and route from the driving simulator. The second stage listens for packets to come through on the created port. Then, based on the choices the user made in the first stage, the program parses the received packet for the variables that were marked to be observed and forwards the results to the third stage. The third stage takes the parsed data and handles the file and network I/O. 


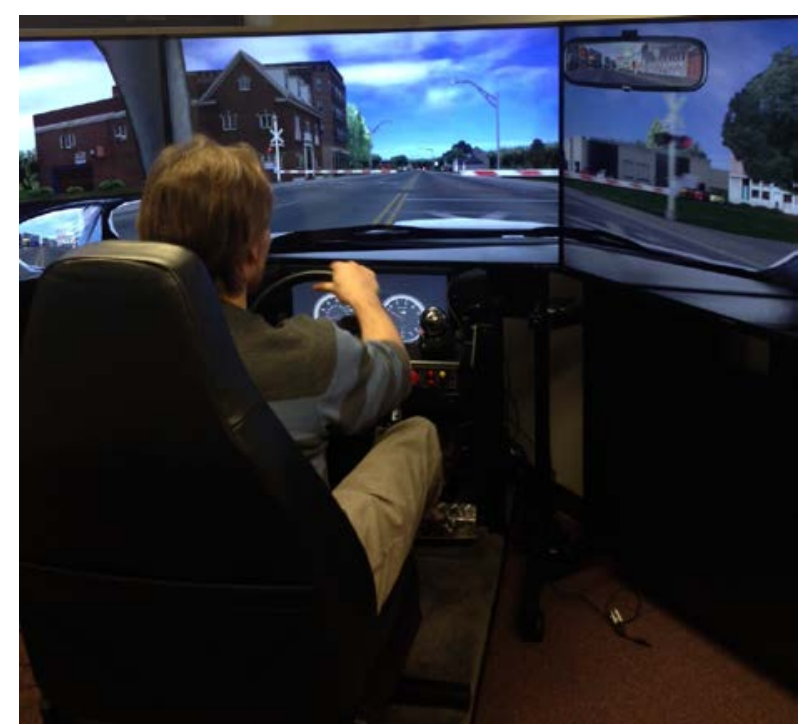

Figure 1. NADS MiniSim simulator as a research platform

\subsection{Data gathering for estimating driver affective states}

The ability to dynamically detect drivers' affective states is crucial in predicting drivers' behavior and performance and guiding them to safer driving. So far, our team has developed a real-time affect detection system using facial expression [7], and empirically tested heart rate [8] and oxygen concentration level of prefrontal lobe of the drivers [9] as an index of affective states (e.g., angry). Our team has intensively used eye-tracking devices to detect a driver's distraction [10], and we also expect that the eye-tracker can detect drivers' cognitive tunneling while they are absorbed in a certain affective state. We would like to extend a range of affective states and appropriate sensors. All these techniques have been used separately. To more effectively estimate drivers' affective states, we plan to integrate all these sensing data by developing data fusion algorithms.

\section{SONIFICATION MAPPING}

This novel system provides researchers with the ability to develop and test auditory displays that reflect situations in the driving simulator in real-time. In the sonification mapping process, we also need to consider other variables, such as human factors and system factors [11] as well as musical parameters (Table 1). For real-time sonification, we use Pure Data (Pd), which is the free real-time graphical dataflow programming environment. Other researchers can also use our Pd patch to test their own driving sonification. The data obtained from the simulator and neurophysiological devices are sent to Pd via Open Sound Control (OSC), which is a commonly used protocol for networking between sound synthesizers and other data.

To make our sonification algorithms, we first define the observation states (i.e., affective states and driving behaviors) and sonification parameters as input measures, and sonification as an output measure. We can define the total observation states function as

ObservationStates $=A S$ (FacialExpression, EyeMovementPattern, HeartRate, Respiration, Skinconductance, BrainWaves, Brainactivities) $x$ DB(LaneDeviation, SteeringWheelAngle, Speed, PedalForce, Collision)
Next, we can define the total sonification parameters function as

SonificationParameters $=M P($ Genre, Key, Tempo, Chord $) \quad x$ $H F$ (Familiarity, Preference, Expectation) x SF(Type, Duration, Timing, Regularity, Interference)

Our goal is to find the best profile of variables for each observation state and then, identify the best profile of sonification parameters to effectively manage driver affective states and driving performance. Therefore, sonification outputs function can be defined as

SonificationOutputs $=f($ ObservationStates $x$ SonificationParameters)

We also plan to expand our system to interact with any number of driving simulators of any level of fidelity and many more physiological sensors and eye trackers. For the applications to real cars, we plan to gather driving data via CAN (controller area network) bus.

Table 1. Mapping variables for observation states and sonification parameters

\begin{tabular}{|c|c|c|}
\hline \multicolumn{2}{|c|}{ Observation States } & $\begin{array}{l}\text { Sonification } \\
\text { Parameters }\end{array}$ \\
\hline Affective States (AS) & $\begin{array}{ll}\text { Driving } & \text { Behaviors } \\
\text { (DB) }\end{array}$ & \\
\hline $\begin{array}{l}\text { - FacialExpression } \\
\text { - EyeMovementPattern } \\
\text { - HeartRate } \\
\text { - Respiration } \\
\text { - SkinConductance } \\
\text { - BrainWaves } \\
\text { - BrainActivities }\end{array}$ & $\begin{array}{l}\text { - LaneDeviation } \\
\text { - SteeringWheelAngle } \\
\text { - Speed } \\
\text { - PedalForce } \\
\text { - Collision }\end{array}$ & $\begin{array}{l}\text { Musical } \\
\text { Parameters } \\
\text { - Genre } \\
\text { - Key } \\
\text { - Tempo } \\
\text { - Chord } \\
\text { Human Factors } \\
\text { - Familiarity } \\
\text { - Preference } \\
\text { - Expectation } \\
\text { System Factors } \\
\text { - Type } \\
\text { - Duration } \\
\text { - Timing } \\
\text { - Regularity } \\
\text { - Interference }\end{array}$ \\
\hline
\end{tabular}

\section{SONIFICATION STRATEGIES}

Researchers in the $1^{\text {st }}$ in-vehicle auditory interaction workshop at ICAD 2015 [12] have discussed different types of sonification strategies appropriate for in-vehicle situations. Based on the discussion, we classify different strategies into three main areas: continuous soundscapes, discrete auditory displays, and target matching auditory displays.

\subsection{Continuous Soundscapes}

Continuous soundscapes are background or atmospheric sounds embedded with information describing mostly low urgency or non-time-sensitive information. Soundscapes allow for a large amount of data to be aggregated and summarized via sound parameter mappings. They have been found to be easily distinguished from background noise, while non-intrusive enough to be easily ignored by the listener when other tasks demand attention [13]. Soundscapes are often reported as informative and relaxing, especially when comprised of natural sound samples (i.e., running water, animal calls, wind rustling tree leaves, etc.). 
For example, certain animal sounds could represent traffic in adjacent lanes. The sound of a swarm of crickets could appear panned and faded in the direction of the vehicle to make the driver aware of the potential obstacle when changing lanes.

\subsection{Discrete Auditory Displays}

Discrete auditory displays come in many forms, but can represent a specific action or event. For example, an earcon representing a takeover requests for a semi-autonomous vehicles would fall into the category of discrete auditory displays. A few ambitious vehicle manufacturers have already introduced lane departure warnings and lane change assistance auditory displays. Before these and other type of hazard detection auditory displays become a standard in all new vehicles, further testing is required to design a system that is both optimally informative and non-intrusive.

\subsection{Target Matching Auditory Displays}

Target matching auditory displays can inform the driver of both the current and optimal state of individual driving tasks. Lane keeping and economical driving are two areas that would benefit from this type of sonification strategy. For instance, the driver's music could be panned to either side to indicate the vehicle's deviation from the center of the lane. The target would be for the driver to keep the music panned to the center indicating that the vehicle is in the proper lateral position (center) of the lane. The music, or any type of audio the driver is listening to, would be panned in the opposite direction of the vehicle's position in the lane. For instance, when the vehicle drifts towards the right side of the lane, the music would be panned to the left, to indicate both the current and target (optimal) lateral lane position. Of course, this can be created based on the principle of gamification.

\section{CONCLUSION \& FUTURE WORKS}

Although our in-vehicle interactive sonification system is in its nascent stage, the potential application for sonification research is immense. Not many driving simulators provide real time data routing for sonification purposes, and even fewer provide that data in an easy to use manner for nonprogrammers. Our software will be further developed to include an easy to use GUI for the user to select variables of interest, and network or port destinations for the data to be channeled to. The integrative data server will be developed to receive, log, and route other data streams from neurophysiological equipment. Considerable interest is directed toward affective computing in the driving domain given the performance deficits of particularly emotional drivers. Future works include a variety of evaluations of different sonification scenarios. Driving performance as well as user acceptance will be collected, as user opinion of the sonification system will be critical for widespread use.

\section{REFERENCES}

[1] J. D. Lee, "Fifty years of driving safety research," Human Factors, vol. 50, no. 3, pp. 521-528, 2008.

[2] A., Jarašūniene, and G., Jakubauskas, "Improvement of road safety using passive and active intelligent vehicle safety systems,” Transport, vol. 22, no. 4, pp. 284-289, 2007.

[3] R., Schoeneburg, and T., Breitling, "Enhancement of active and passive safety by future PRE-SAFE systems," In 2005 Conf. on ESV, Washington DC, pp. 05-0080, 2005.

[4] C. D., Wickens, "Multiple resources and performance prediction,” Theoretical Issues in Ergonomics Science, vol. 3, pp. 159-177, 2002.

[5] T., Kramer, "An introduction to auditory display," Auditory Display: Sonification, Audification, and Auditory Interfaces, In G. Kramer (Ed.), AddisonWesley, 1-77, 1992.

[6] S. FakhrHosseini, P., Kirby, and M., Jeon, "Regulating drivers' aggressiveness by sonifying emotional data. In Proc. of the 21st Int. Conf. (ICAD 2015). New York, USA, July 2015.

[7] M. Jeon, and B. N. Walker, "Emotion detection and regulation interface for drivers with traumatic brain injury,” In Proc. of the Int. Conf. (CHI'11), Diversity Workshop, Vancouver, BC, Canada, May 7-12, 2011.

[8] S. Jansen, A. Westphal, M. Jeon, and, A. Riener, "Detection of drivers' incidental and integral affect using physiological measures," In Adjunct Proc. of the 5th Int. Conf. (AutomotiveUI'13), pp. 97-98, Eindhoven, The Netherlands, October 27-30, 2013.

[9] S. M. FakhrHosseini, M. Jeon, and R. Bose, "Estimation of drivers' emotional states based on neuroergonomic equipment: An exploratory study using fNIRS,” In Proc. of the 7th Int. Conf. (AutomotiveUI'15), Nottingham, UK, September 1-3, 2015.

[10] S. M., Fakhrhosseini, M. Jeon, P. Lautala, and D. Nelson, "An investigation on driver behaviors and eyemovement patterns at grade crossings," In. Proc. of the Joint Rail Conf. (JRC2015), San Jose, CA, March 23-26, 2015.

[11] M. Jeon, "A systematic approach to using music for mitigating affective effects on driving performance and safety," In Proc. of the 14th ACM Int. Conf. (UbiComp'12), ACM Press, pp. 1127-1132, Pittsburgh, USA, September 5-8, 2012.

[12] M. Jeon et al., "Proceedings of the in-vehicle auditory interactions workshop," In Proc. of the 21st International Conference on Auditory Display (ICAD2015), Graz, Austria, July 8-10, 2015.

[13] K. Wolf, G. Gliner, and R. Fiebrink, "A model for datadriven sonification using soundscapes," In Proc. of the 20th Int. Conf. (IUIC), ACM Press, 2015. 